\title{
Notes on Some Plant Collections from Bachok and Several Forest Reserves in Kelantan
}

\author{
Lim A.L., Haron N.W., Yong K.T. and M.M. Wong. \\ Institute of Biological Sciences, Faculty of Science, University of Malaya, 50603 Kuala Lumpur, Malaysia. \\ *ahlan@um.edu.my (corresponding author) \\ Received on $6^{\text {th }}$ October 2009, accepted in revised form $14^{\text {th }}$ December 2009.
}

\begin{abstract}
A botanical survey of Bachok and various forest reserves at Jeram Linang, Jeram Pasu and Bukit Bakar was part of an expedition from 14-20 June 2008 carried out by the IOES (Institute of Ocean and Earth Sciences) University of Malaya to survey and prepare an inventory of the biodiversity of the coastal region around Bachok, Kelantan. A total of 54 species from 30 families, consisting of seashore and mangrove plants were identified from the coastal area of Bachok and Semerak; while 89 species of flowering plants representing 44 families, a single gymnosperm, Agathis borneensis (Araucariaceae); and 15 genera and 23 species of mosses (from 10 families) were recorded in the three forest reserves. Several of the species collected were rarely found outside Kelantan.
\end{abstract}

ABSTRAK Satu survei tumbuhan di Bachok dan beberapa hutan simpanan termasuk Jeram Linang, Jeram Pasu dan Bukit Bakar, Kelantan adalah sebahagian ekspedisi survei biodiversiti kawasan pantai di sekitar Bachok Kelantan oleh IOES (Institute of Ocean and Earth Sciences) Universiti Malaya pada 14-20 Jun 2008. Sejumlah 54 spesies daripada 30 famili tumbuhan pantai dan bakau dikenalpasti dari kawasan pantai Bachok dan Semerak; sedangkan 89 spesies tumbuhan berbunga (mewakili 44 famili), satu gimnosperma, Agathis borneensis (Araucariaceae); serta 15 genera dan 23 spesies daripada 10 famili lumut sejati telah direkodkan di kawasan sekitar Jeram Linang, Jeram Pasu dan Hutan Lipur Bukit Bakar. Beberapa spesies yang dikutip adalah jarang ditemui di luar Kelantan.

(Keywords: botanical survey, angiosperms, mosses, Kelantan)

\section{INTRODUCTION}

Kelantan $\left(101^{\circ} 20^{\prime} \mathrm{E}\right.$ to $102^{\circ} 40^{\prime} \mathrm{E}$ and $04^{\circ} 30^{\prime} \mathrm{N}$ to $\left.06^{\circ} 15^{\prime} \mathrm{N}\right)$ in northeast Peninsular Malaysia has an area of 1.5 million hectares of which $50 \%$ is under forest and wildlife reserves [1]. In Peninsular Malaysia it is the state with the largest area of reserved forest. Its landscape is extremely diverse, ranging from seashores, mangroves and flat plains in the east gradually rising (westwards) towards hilly and mountainous terrain drained by several river systems. Thus the vegetation varies from seashore vegetation and mangrove along the coast, to the extremely species-rich Tropical Evergreen Lowland Rainforest; modified by the influx of monsoon elements from Burma and Siam [2, 3, and 4].

Bachok (25 km east of Kota Bharu) and Semerak (in Pasir Puteh, $50 \mathrm{~km}$ southeast of Kota Bharu) are popular recreational destinations owing to their famous scenic sandy beaches. Jeram Linang and Bukit Bakar (Machang district) and Jeram Pasu (Pasir Putih) are the earliest waterfalls in Kelantan to be developed with basic amenities (such as chalets, public toilets, car parks, restaurants, camping sites, jungle treks etc) for recreational activities. These are popular recreation sites for the local people and contribute to increasing public awareness of the importance of forests for people. On the other hand, and as elsewhere, Kelantan's forest reserves also encounter illegal encroachment and taking of forest products. These and forest conversion can lead to rapid depletion of native species. In that context, the present survey is a small study undertaken within a short time; however, various interesting aspects are presented as contribution towards the documentation of Kelantan's flora for conservation purposes.

\section{MATERIALS AND METHODS}

This botanical survey was conducted from 14-20 June 2008. Specimens were collected in UM land, Bachok (N 06 00' 25", E $\left.102^{\circ} 25^{\prime} 37^{\prime \prime}\right)$; Semerak (N $\left.05^{\circ} 52^{\prime} 18^{\prime \prime}, \mathrm{E} 102^{\circ} 30^{\prime} 41^{\prime \prime}\right)$; the Forest Reserve areas at Jeram Linang, Machang ( $\mathrm{N} 05^{\circ} 44^{\prime} 27^{\prime \prime}, \mathrm{E} 102^{\circ} 22^{\prime}$ $36^{\prime \prime}$ ); Jeram Pasu, Pasir Puteh (N 05 47' 63", E $102^{\circ}$ $\left.20^{\prime} 21^{\prime \prime}\right)$; and Bukit Bakar Hutan Simpanan Ulu Sat, Machang (N 05 43' 1", E $102^{\circ} 16^{\prime} 38^{\prime \prime}$ ). Moss specimens growing on soil, rocks, tree branches, trunks and exposed roots, rotten logs and wood were 
collected and identified in the laboratory. Angiosperm specimens of flower and fruit were identified in the field or collected, tagged and brought back to the laboratory for further identification. Herbarium specimens were prepared and kept in the University of Malaya Herbarium (KLU).

\section{RESULTS AND DISCUSSION}

Angiosperm and moss species collected are given in Tables 1-3. The coastal areas of Bachok and Semerak recorded 54 species from 30 families, comprising typical seashore vegetation (Table 1) such as Canavalia rosea, Casuarina equisetifolia, Ipomoea pes-caprae, Pandanus odoratissimus, Vitex odorata, Hibiscus tiliaceus and Spinifex littoreus which are common in the sandy beaches of Kelantan. Further inland, Catunaregam tomentosa is one of the most common shrub species observed. Besides seashore vegetation, the Semerak area also has pockets of mangrove along the Semerak river. The mangrove identified included species such as Rhizophora apiculata, R. mucronata, Avicennia alba, Ceriops tagal, Sonneratia alba, Brugurira cylindrica, Lumnitzera racemosa, as well as the less common Cayratia trifolia and Derris heptaphyla.

A total of 44 families of angiosperms comprising 89 species were recorded (Table 2) and 10 families of mosses made up of 15 genera and 23 species were collected from areas around the three forest reserves. These study sites are well irrigated by swift-flowing streams, often cascading down boulders as waterfalls, creating a range of niches for plants. On large shaded, damp boulders and tree branches and trunks are found 15 genera of mosses from 10 families (Table 3 ). The most common genus is Calymperes (family Calymperaceae) collected from both Jeram Linang and Bukit Bakar. Of the 23 species collected, an epiphyllous moss, Aerobropsis subleptostigmata (family Meteoriaceae) is a new record for Kelantan since this species has only been collected before from two locations, i.e. Taman Negara (Yong, unpublished data) and Cameron Highlands $[5,6]$.

At Jeram Linang and Jeram Pasu (below $300 \mathrm{~m}$ elevation) and Bukit Bakar (400-500 m), the natural vegetation is Mixed Dipterocarp Forest with a good representation of the Dipterocarpaceae, Rubiaceae, Euphorbiaceae, Fabaceae, Melastomataceae and Arecaceae (see Table 2) among 'standard' lowland forest angiosperm families of Peninsular Malaysia [7]. Although this quick survey was to document genera that could be identified by flowers and fruits, not many species of such genera were reproductive at the same time, thus the number of species listed in Table 2 does not reflect the actual richness of a particular genus such as in the Dipterocarpaceae (Shorea and Dipterocarpus), Moraceae (Artocarpus and Ficus) and Rubiaceae (Psychotria and Chasalia).

In the many forest gaps are found the pioneer and secondary forest species such as macarangas, melastoma, bamboos, grasses and ferns. Clidemia hirta, a highly invasive plant in tropical forests [8] has also been collected. Nevertheless, this present study reveals that these forest reserves still harbour some very unique and potentially valuable species including Molineria latifolia and Eurycoma, both threatened species useful in therapeutics; Zanthoxylum rhetsa, practically endemic to Kelantan, Psychotria a few species of this genus are very much sought after by herbalists as a pain killer and anticancer plant and many other medicinal species such as Ficus, Chasalia, Alstonia, Calophyllum and others. Agathis borneensis very commonly observed in Bukit Bakar probably owing to its sandy soil and slightly higher elevation, is not seen in Jeram Linang and Jeram Pasu.

It is estimated that $12,000-15,000$ seed plant species are found in this region, of which about $20 \%$ are endemic [9]. Many of these species are known medicinal plants and are commonly used in the daily life of the local inhabitants for the treatment of various ailments especially for skin complaints and diseases of womenfolk and recuperation after childbirth [10]. Traditionally, the forests yield not only timbers but also a host of other non timber products such as fruits and vegetables, latex, dyes, resins, herbal medicines, ornamentals, etc. [11]. As there is no national list of threatened and endangered plant species [9], the conservation status of our flora is uncertain and many of these plants together with their natural habitats could be lost forever (e.g. Nepenthes benstonei endemic to this region [12] was not seen in this study).

Despite the short survey, several interesting elements of the flora were detected (new record of epiphyllous moss, Aerobropsis subleptostigmata, detection of invasive Clidemia hirta, collection of threatened species such as Eurycoma and Molineria latifolia) indicating that more concerted efforts at documentation of this region would certainly be very instructive and yield invaluable and interesting information. 
Table 1. List of seed plants recorded from UM land (U) in Bachok and Semerak (S).

\begin{tabular}{|c|c|c|}
\hline Family & Genus / Species & Locations \\
\hline \multicolumn{3}{|c|}{ Magnoliophyta (flowering plants) } \\
\hline \multirow[t]{2}{*}{ Acanthaceae } & Asystasia intrusa Blume & $\mathrm{U}$ \\
\hline & Avicennia alba Blume & $\mathrm{S}$ \\
\hline Aizoaceae & Sesuvium portulacastrum (L.) L. & $\mathrm{S}$ \\
\hline Amaryllidaceae & Crinum asiaticum $\mathrm{L}$. & $\mathrm{U}$ \\
\hline Apocynaceae & Alstonia angustifolia A. DC. & $\mathrm{S}$ \\
\hline Arecaceae & Nypa fruticans Wurmb. & $\mathrm{U}, \mathrm{S}$ \\
\hline \multirow{2}{*}{ Asteraceae } & Emilia sonchifolia (L.) DC. ex Wight & $\mathrm{U}$ \\
\hline & Wedelia biflora (L.) DC. & $\mathrm{U}, \mathrm{S}$ \\
\hline Cleomaceae & Cleome sp. & $\mathrm{S}$ \\
\hline Casuarinaceae & Casuarina equisetifolia Blanco & $\mathrm{U}, \mathrm{S}$ \\
\hline Clusiaceae & Calophyllum inophyllum L. & $\mathrm{U}$ \\
\hline \multirow{2}{*}{ Combretaceae } & Lumnitzera racemosa Willd. & $\mathrm{S}$ \\
\hline & Terminalia catappa $\mathrm{L}$. & $\mathrm{U}, \mathrm{S}$ \\
\hline Convolvulaceae & Ipomoea pes-caprae (L.) R. Br. & $\mathrm{U}, \mathrm{S}$ \\
\hline Cyperaceae & Cyperus sp. & $\mathrm{U}$ \\
\hline \multirow[t]{2}{*}{ Dilleniaceae } & Tetracera scandens Merrill & $\mathrm{S}$ \\
\hline & Tetracera indica Merrill & $\mathrm{S}$ \\
\hline Euphorbiaceae & Excoecaria agallocha $\mathrm{L}$. & $\mathrm{S}$ \\
\hline \multirow{5}{*}{ Fabaceae } & Canavalia rosea (Sw.) DC. & $\mathrm{U}$ \\
\hline & Cassia sp. & $\mathrm{S}$ \\
\hline & Derris heptaphylla Merrill & $\mathrm{S}$ \\
\hline & Desmodium laxum DC. & $\mathrm{S}$ \\
\hline & Indigofera hirsuta Harv. & $\mathrm{U}$ \\
\hline \multirow{4}{*}{ Lamiaceae } & Clerodendrum inerme (L.) Gaertn. & $\mathrm{S}$ \\
\hline & Premna tomentosa Kurz & $\mathrm{S}$ \\
\hline & Vitex ovata C.P. Thunberg ex A. Murray & $\mathrm{U}, \mathrm{S}$ \\
\hline & Vitex pinnata $\mathrm{L}$ & $\mathrm{S}$ \\
\hline Lythraceae & Sonneratia alba Griff. & $\mathrm{S}$ \\
\hline \multirow[t]{3}{*}{ Malvaceae } & Abutilon sp. & $\mathrm{U}$ \\
\hline & Hibiscus tiliaceus L. & $\mathrm{U}, \mathrm{S}$ \\
\hline & Urena lobata $\mathrm{L}$. & $\mathrm{U}$ \\
\hline Moraceae & Ficus superba (Miq.) Miq. & $\mathrm{S}$ \\
\hline Myrtaceae & Eugenia grandis Wight & $\mathrm{S}$ \\
\hline Pandanaceae & Pandanus odoratissimus Blume & $\mathrm{U}, \mathrm{S}$ \\
\hline \multirow[t]{2}{*}{ Phyllanthaceae } & Glochidion sp. & $\mathrm{S}$ \\
\hline & Phyllanthus pulcher Wall. & $\mathrm{U}$ \\
\hline \multirow[t]{2}{*}{ Poaceae } & Ischaemum muticum $\mathrm{L}$. & $\mathrm{U}$ \\
\hline & Spinifex littoreus Merrill & $\mathrm{U}, \mathrm{S}$ \\
\hline \multirow[t]{5}{*}{ Rhizophoraceae } & Bruguiera cylindrica (L.) Blume & $\mathrm{S}$ \\
\hline & Ceriops tagal (Perr.) C.B. Rob. & $\mathrm{S}$ \\
\hline & Rhizophora apiculata Blume & $\mathrm{S}$ \\
\hline & Rhizophora mucronata Lam. & $\mathrm{S}$ \\
\hline & Rhizophora stylosa Griff. & $\mathrm{S}$ \\
\hline \multirow[t]{6}{*}{ Rubiaceae } & Borreria hispida K. Schum. & $\mathrm{U}$ \\
\hline & Catunaregam tomentosa-(B1. Ex DC.) D.D. Tirvengadum & $\mathrm{S}$ \\
\hline & Guettarda speciosa $\mathrm{L}$. & $\mathrm{S}$ \\
\hline & Hedyotis corymbosa (L.) Lam. & $\mathrm{U}$ \\
\hline & Morinda citrifolia Linn. & $\mathrm{S}$ \\
\hline & Uncaria cordata (Lour.) Merr. & $\mathrm{U}$ \\
\hline Rutaceae & Melicope sp. & $\mathrm{S}$ \\
\hline Sapindaceae & Sapindus sp. & $\mathrm{S}$ \\
\hline Smilacaceae & Smilax sp. & $\mathrm{S}$ \\
\hline Taccaceae & Tacca integrifolia Ham.ex Hook.f. & $\mathrm{U}$ \\
\hline Vitaceae & Cayratia trifolia (L.) Domin & $\mathrm{S}$ \\
\hline
\end{tabular}


Table 2. List of seed plants recorded from the Forest Reserves of Jeram Linang (L), Jeram Pasu (P) and Hutan Lipur Bukit Bakar (B).

\begin{tabular}{lcc}
\hline Family & Genus / Species & Locations \\
\hline Magnoliophyta (flowering plants) &
\end{tabular}

Acanthaceae

Agavaceae

Anacardiaceae

Anisophylleaceae

Apocynaceae

Araceae

Araliaceae

Arecaceae

Arecaceae

Brownlowiaceae

Burseraceae

Clusiaceae

Dilleniaceae

Dipterocarpaceae

Ebenaceae

Erythropalaceae

Euphorbiaceae

Fabaceae

Fagaceae

Gentianaceae

Hypericaceae

Hypoxidaceae
Justicia sp. $\quad$ B

Dracaena $\mathrm{sp}$.

Pentaspadon velutinus Hook.f. L

Swintonia sp.

Campnosperma macrophylla Hook.f.

Bouea macrophylla Griff.

Anisophyllea sp.

Alstonia angustifolia A. DC.

Alstonia angustiloba Miq.

Dischidia sp.

Tabernaemontana sp.

Amorphophallus sp.

Pothos sp.

Arthrophyllum diversifolium Blume L, B

Areca sp.

Arenga sp.

Calamus sp.

Caryota mitis Herb.

Eugeisonna tristis Griff.

Licuala sp.

Pinanga sp.

Salacca sp.

Pentace sp.

Pentace macrophylla King

Canarium sp.

Calophyllum sp.

Garcinia nervosa Miq.

Tetracera sp.

Cotylelobium sp.

Dipterocarpus sp.

Hopea odorata Roxb.

Parashorea stellata Kurz

Shorea guiso Blume

Shorea leprosula Miq.

Shorea sp.

Diospyros buxifolia Hiern

Strombosia javanica Blume L

Elateriospermum sp.

Endospermum sp.

Macaranga gigantean Muell. Arg.

Macaranga javanica Muell. Arg.

Macaranga motleyana Muell. Arg.

Macaranga triloba Muell. Arg.

Sapium baccatum Roxb.

Intsia palembanica Miq.

Koompasia malaccensis Maing.

Archidendron jiringa (Jack) I. Nielsen

Uraria crinite (L.)DC.

Lithocarpus sp.

Fagraea racemosa Jack ex Wallich

Cratoxylum cochinchinense (Lour.) B1.

Molineria latifolia Herb. ex Kurz
L

$\mathrm{P}$

B

L, B

L

L

$\mathrm{P}$

$\mathrm{P}$

L

L

L

L

$P$

L, P

L, B

L, P, B

B

B

L

L

L

L

L

P

L

B

L

L

L

L, B

B

B

B

B

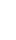

$$
\text { (1) }
$$$$
\text { (n) }
$$$$
\text { ( }
$$

L

L

B

L

L

L

L

L

L

L

L

L

L

B

L

P

L

L, P 


\begin{tabular}{|c|c|c|}
\hline \multirow[t]{2}{*}{ Lauraceae } & Phoebe grandis (Nees) Merrill & B \\
\hline & Cinnamomum sp. & $\mathrm{L}, \mathrm{B}$ \\
\hline Lecythidaceae & Barringtonia sp. & $\mathrm{L}$ \\
\hline Malvaceae & Durio sp. & $\mathrm{L}, \mathrm{P}$ \\
\hline \multirow[t]{5}{*}{ Melastomataceae } & Clidemia hirta D. Don & $\mathrm{L}$ \\
\hline & Lijndenia laurina Zoll. \& Mor. & $\mathrm{B}$ \\
\hline & Melastoma malabathricum Jack & $\mathrm{P}$ \\
\hline & Memecylon sp. & $\mathrm{L}$ \\
\hline & Pternandra sp. & $\mathrm{B}$ \\
\hline Meliaceae & Sandoricum koetjape Merrill & $\mathrm{L}$ \\
\hline \multirow[t]{2}{*}{ Moraceae } & Artocarpus altilis (Parkinson) Fosberg & $\mathrm{L}$ \\
\hline & Ficus sp. & $\mathrm{L}$ \\
\hline Musaceae & Musa sp. & $\mathrm{B}$ \\
\hline Myristicaceae & Knema. sp. & $\mathrm{L}$ \\
\hline \multirow[t]{2}{*}{ Myrsinaceae } & Ardisia crenata Roxb. & $\mathrm{L}, \mathrm{B}$ \\
\hline & Labisia $\mathrm{sp}$ & B \\
\hline \multirow{2}{*}{ Myrtaceae } & Eugenia grandis Wight & $\mathrm{L}$ \\
\hline & Rhodamnia cinerea Jack & $\mathrm{P}$ \\
\hline Ochnaceae & Brackenridgea palustris Bartell. & $\mathrm{L}$ \\
\hline Pandanaceae & Pandanus sp. & $\mathrm{L}, \mathrm{P}, \mathrm{B}$ \\
\hline Pentaphragmataceae & Pentaphragma sp. & B \\
\hline Phyllanthaceae & Antidesma cuspidatum Muell. Arg. & $\mathrm{L}, \mathrm{B}$ \\
\hline Polygalaceae & Xanthophyllum sp. & B \\
\hline \multirow{7}{*}{ Rubiaceae } & Chassalia sp. & $\mathrm{L}$ \\
\hline & Ixora sp. & $\mathrm{L}, \mathrm{P}, \mathrm{B}$ \\
\hline & Psychotria sp. & $\mathrm{L}, \mathrm{B}$ \\
\hline & Rennellia paniculata King \& Gamble & $\mathrm{L}$ \\
\hline & Rennellia speciosa Benth. \& Hook.f. & $\mathrm{B}$ \\
\hline & Tarenna fragrans Koord. \& Valet. & $\mathrm{L}$ \\
\hline & Uncaria sp. & $\mathrm{L}$ \\
\hline Rutaceae & Zanthoxylum rhetsa DC. & $\mathrm{L}$ \\
\hline Sapindaceae & Nephelium sp. & $\mathrm{L}$ \\
\hline Simaroubaceae & Eurycoma longifolia Jack & $\mathrm{B}$ \\
\hline Smilacaceae & Smilax sp. & $\mathrm{L}, \mathrm{B}$ \\
\hline Thymelaeaceae & Gonystylus bancanus (Miq.) Kurz & $\mathrm{B}$ \\
\hline \multirow[t]{2}{*}{ Zingiberaceae } & Alpinia sp. & $\mathrm{L}$ \\
\hline & Etlingera $\mathrm{sp}$. & B \\
\hline \multicolumn{3}{|l|}{ Pinophyta } \\
\hline Araucariaceae & Agathis borneensis Warburg & $\mathrm{B}$ \\
\hline
\end{tabular}


Table 3. List of mosses collected from the Forest Reserves of Jeram Linang (L), Jeram Pasu (P) and Hutan Lipur Bukit Bakar (B).

\begin{tabular}{|c|c|c|}
\hline Family & Genus / Species & Locations \\
\hline \multirow{9}{*}{ Calymperaceae } & Calymperes erosum Müll. Hal. & $\mathrm{L}, \mathrm{B}$ \\
\hline & Calymperes lonchophyllum Schwägr. & $\mathrm{L}$ \\
\hline & Calymperes moluccense Schwägr. & $\mathrm{L}$ \\
\hline & Calymperes porrectum Mitt. & $\mathrm{L}$ \\
\hline & Calymperes tahitense (Sull.) Mitt. & $\mathrm{L}, \mathrm{B}$ \\
\hline & Mitthyridium flavum (Müll. Hal.) H. Rob. & $\mathrm{P}$ \\
\hline & Mitthyridium obtusifolium (Lindb.) H. Rob. & $\mathrm{B}$ \\
\hline & Mitthyridium repens (Harv.) H. Rob. & $\mathrm{L}$ \\
\hline & Syrrhopodon spiculosus Hook. \& Grev. & $\mathrm{B}$ \\
\hline Fissidentaceae & Fissidens crispulus Brid. & $\mathrm{L}$ \\
\hline \multirow[t]{3}{*}{ Hypnaceae } & Ectropothecium sp. & $\mathrm{L}$ \\
\hline & Ectropothecium zollingeri (Müll. Hal.) A. Jaeger & $\mathrm{P}$ \\
\hline & Vesicularia dubyana (Müll. Hal.) Broth. & $\mathrm{L}$ \\
\hline Leucobryaceae & Leucobryum sanctum (Nees ex Schwägr.) Hampe & $\mathrm{L}$ \\
\hline Meteoriaceae & Aerobropsis subleptostigmata Broth. \& Paris & $\mathrm{P}$ \\
\hline Neckeraceae & Pinnatella mucronata (Bosch \& Sande Lac.) M. Fleisch. & $\mathrm{L}$ \\
\hline Phyllodrepaniaceae & Mniomalia semilimbata (Mitt) Müll. Hal. & $\mathrm{L}$ \\
\hline Pterobryaceae & Oedicladium pseudorufescens (Hampe) B.C. Tan \& Mohamed & $\mathrm{L}$ \\
\hline \multirow{4}{*}{ Sematophyllaceae } & Taxithelium nepalense (Schwägr) Broth. & $\mathrm{L}, \mathrm{B}$ \\
\hline & Trichosteleum boschii (Dozy \& Molk.) A. Jaeger & $\mathrm{B}$ \\
\hline & Trichosteleum stigmosum Mitt. & $\mathrm{B}$ \\
\hline & Trismegistia lancifolia(Harv.) Broth. & $\mathrm{P}, \mathrm{L}$ \\
\hline Thuidiaceae & Thuidium pristocalyx (Müll. Hal.) A. Jaeger & $\mathrm{L}$ \\
\hline
\end{tabular}

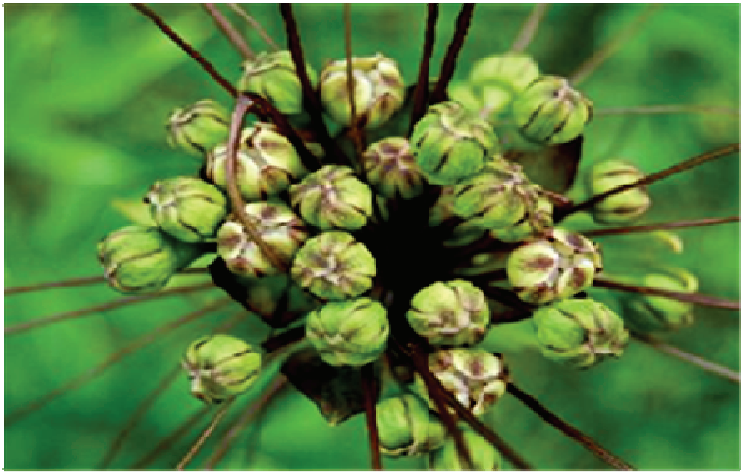

A. Tacca integrifolia

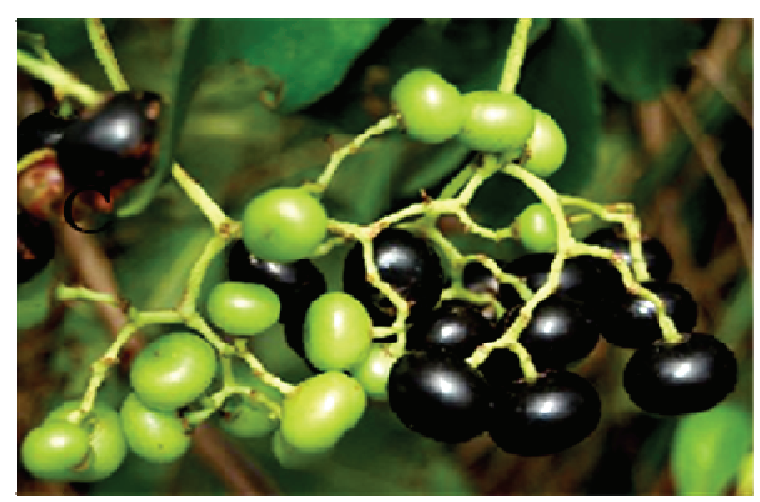

C. Cayratia trifolia

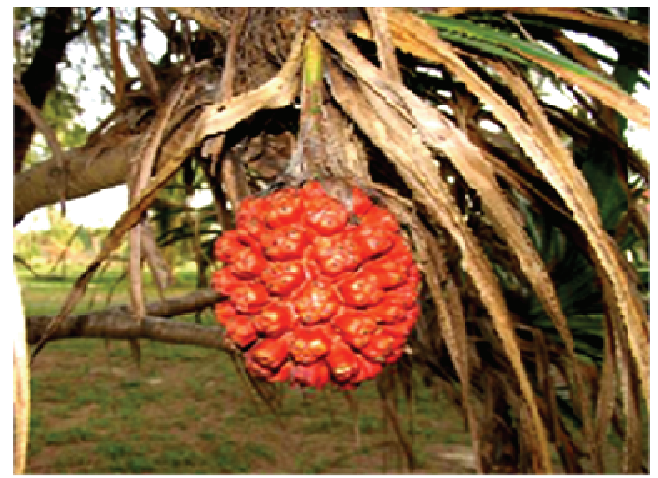

B. Pandanus odoratissimus

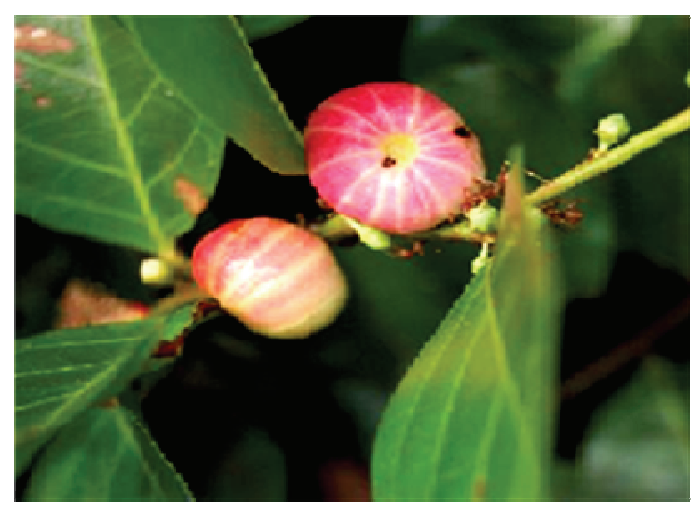

D. Glochidion sp. 


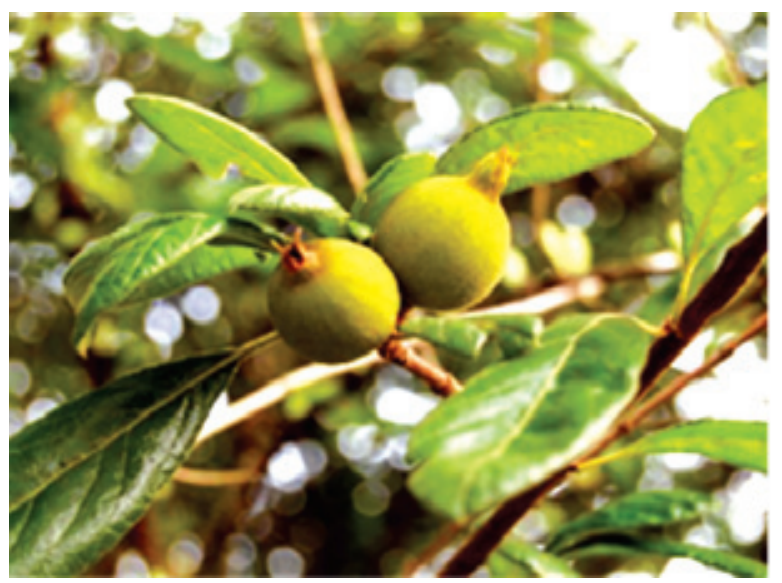

E. Catunaregam tomentosa - fruit

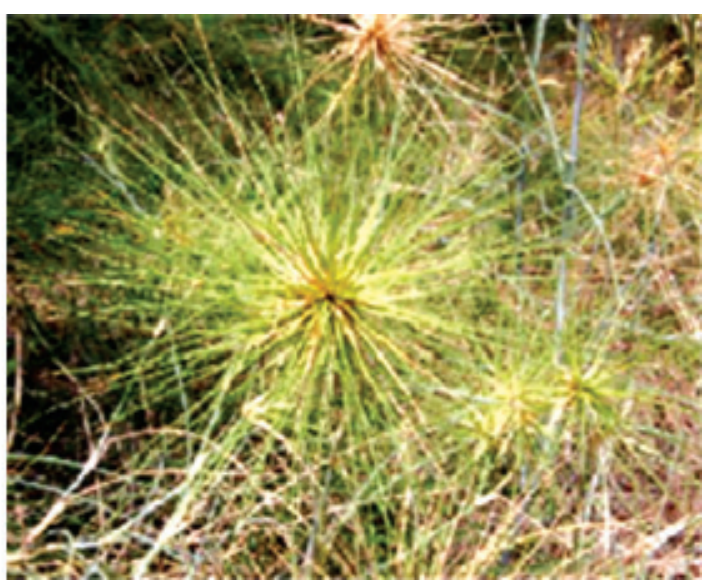

F. Spinifex littoreus - fruit

Figure 1. A. Tacca integrifolia, B. Pandanus odoratissimus, C. Cayratia trifolia, D. Glochidion sp., E. Catunaregam tomentosa - fruit, F. Spinifex littoreus - fruit.

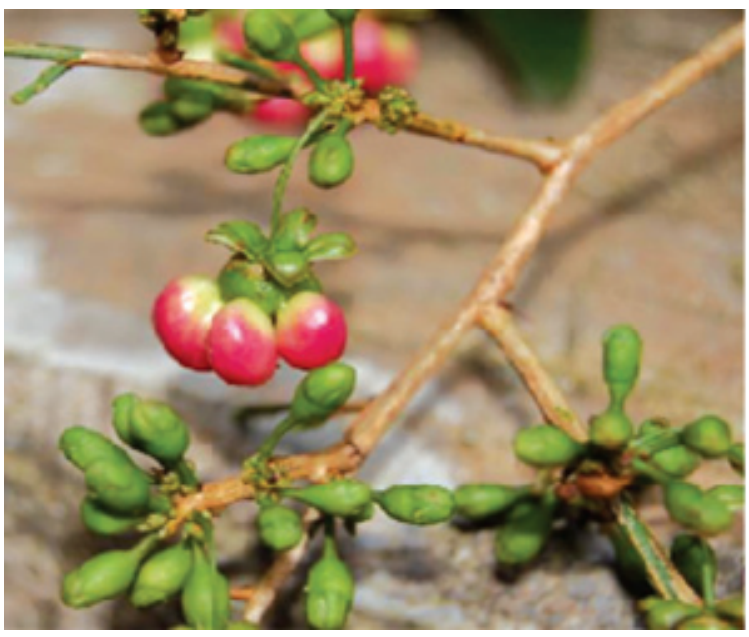

A. Brackenridgea palustris

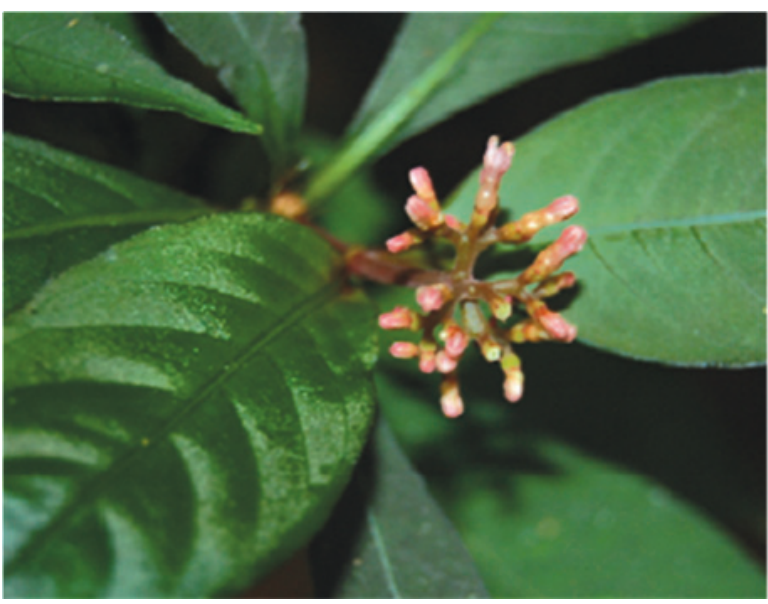

C. Chassalia sp.

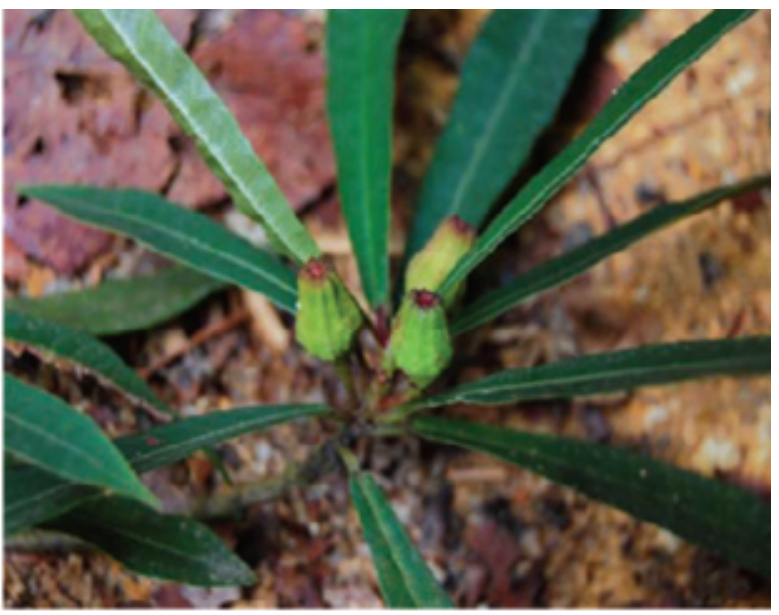

B. Ficus sp.

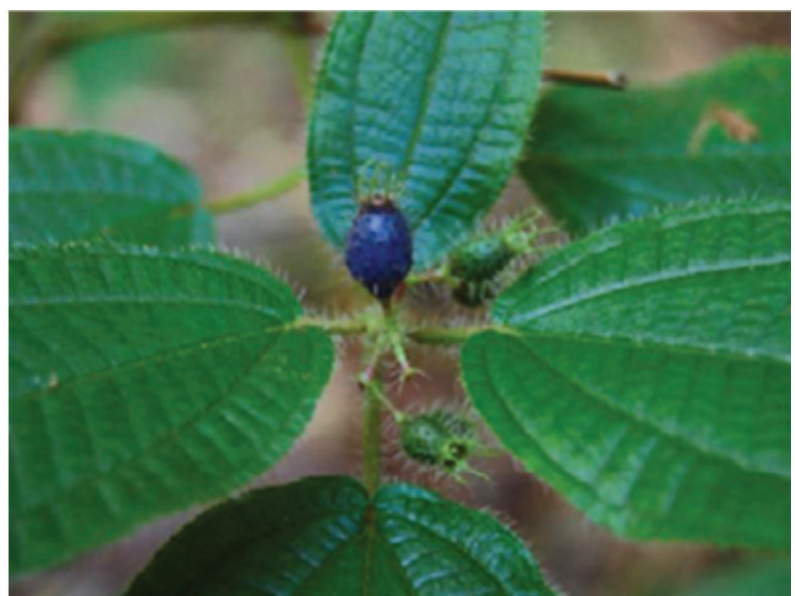

D. Clidemia hirta

Figure 2. A. Brackenridgea palustris, B. Ficus sp., C. Chasalia sp., D. Clidemia hirta 


\section{ACKNOWLEDGEMENTS}

Financial assistance from the University of Malaya (FS290/2008A) for this study is gratefully acknowledged. We thank Professor Dr Wong Khoon Meng for help in identifying several specimens collected; Professor Dr. Chong Ving Ching, Dr. Sasekumar, Encik Zulkapli Ibrahim, Encik Anuar and Cik Shazlizawati from University of Malaya and staff of Kelantan Forestry Department for their assistance during field work.

\section{REFERENCES}

1. Kamaruzaman Jusoff \& Skidmore, A. K. 2009. Geoinofrmation science for sustainable development of Mount Stong F.R., Kelantan, Malaysia. Journal of Sustainable Development 2(1): 51-64.

2. Corner, E.J.H. 1952. Wayside Trees of Malaya, Vol. 1. Second Edition. Government Printer, Singapore.

3. Whitmore, T.C. 1975. Tropical Rain Forests of the Far East. Clarendon Press, Oxford and London.

4. Yamada I. 1997. Tropical Rainforest of Southeast Asia. University of Hawaii Press, Honolulu.

5. Noguchi, A. 1973. Contributions to the bryology of Thailand. J. Hattori Bot. Lab. 37: 235-250.

6. Noguchi, A. 1976. A taxonomic revision of the family Meteoriaceae of Asia. J. Hattori Bot. Lab. 41: 231-357.

7. Kochummen, K.M., LaFrankie, J.V., and Manokaran, N. 1990. Floristic composition of Pasoh Forest Reserve, a lowland rain forest in Peninsular Malaysia. J. Trop. For. Sci. 3: 1 -13.

8. Peters, H.A. 2001. Clidemia hirta invasion at the Pasoh Forest Reserve: an unexpected plant invasion in an undisturbed tropical forest. Biotropica 33(1): 60-68.

9. Saw, L.G., Chua, L.S.L. \& Abdul Rahman, N. (Eds) 2009. Malaysia. National Strategy for Plant Conservation. Ministry of Natural Resources and Environment and Forest Research Institute Malaysia.

10. Ong, H.C. \& Noridiana M. 1999. Malay ethnomedico botany in Machang, Kelantan Malaysia. Fitoterapia 70 (5): 502-513.

11. Pei S.J. 2007. Traditional knowledge and sustainable use of plant resources: ethnobotanical indicators of plant use. International Forum on Protecting Intellectual Property Rights (IPRs) of Traditional Knowledge and Sustainable Development, Aug 12-13, 2007, Kunming, Yunan, China.
12. Clarke, C.M. 1999. Nepenthes benstonei (Nepenthaceae), a new pitcher plant from Peninsular Malaysia. Sandakania 13: 79-87. 